\title{
Changes in Emergency Department Responses Between SARS and COVID-19 in Taiwan
}

Chia Wei Lin

China Medical University https://orcid.org/0000-0002-9380-8337

Kuan-Ho Lin

China Medical University Hospital

Hong-Mo Shih

China Medical College: China Medical University

Kai-Wei Yang

China Medical University Hospital

Kao-Pin Hwang

China Medical University Hospital

Wei-Kung Chen ( $\nabla$ ercwk@mail.cmuh.org.tw )

Emergency Department, China Medical University Hospital, Taiwan

\section{Research}

Keywords: SARS outbreak, emergency department (ED), COVID-19 epidemic

Posted Date: November 17th, 2020

DOI: https://doi.org/10.21203/rs.3.rs-106038/v1

License: @ (i) This work is licensed under a Creative Commons Attribution 4.0 International License. Read Full License 


\section{Abstract}

Background: After SARS outbreak, infectious control polices had been reformed in Taiwan, but there was no evidence to prove its effectiveness. This study compared emergency department (ED) responses to the SARS and COVID-19 epidemics and investigate how policy changes affect infection prevention.

Methods: A 2003 questionnaire regarding the responses of EDs to SARS was resent to EDs during the COVID-19 epidemic in 2020. The use of personal protective equipment (PPE), implementation of infection control measures (ICMs), and difficulties in performance were compared. Data collection included hospital level, different PPE types provided and ICMs implemented, timing for using PPE and ICMs, and a difficulty rating scale for ICM implementation.

Results: In total, 100 EDs responded to the questionnaire in 2003 was reviewed and compared with 131 EDs in 2020. In COVID-19 epidemic, the use of basic PPEs and ICMs was mostly significantly improved, but the percentage of preparedness in high grade PPEs was still low. Quarantine of fever patients outside of EDs was less performed in small to medium sized hospitals ( $p=0.008$ and 0.004). All of the additional ICMs were significantly less implemented in local hospitals. The timing to use PPE and implement ICMs were simultaneously and significantly performed at early stage. Instituting a fever triage ward and restricting fever patient admission became less necessary. The closure of EDs remained the most difficult to perform in both outbreaks.

Conclusion: After the policy reforms, ED responses became earlier and more consistent. However, inadequate quarantine resources at EDs in low- and middle-grade hospitals may lead to breaches in future epidemics.

\section{Contributions To The Literature}

Taiwan's success in preventing COVID-19 spread is obvious all over the world.

This study helps to find out the key to the success of frontline personnel in epidemic prevention form the perspective of emergency department.

The crisis that may be encountered in the face of epidemic prevention was also noted in the study.

\section{Introduction}

Coronavirus disease 2019 (COVID-19) pandemic was the third major pandemic of the 21st century after severe acute respiratory syndrome (SARS) and Middle East respiratory syndrome (MERS) pandemics. SARS coronavirus was transmitted by humans through 26 countries, with more than 8000 people affected, in 2003[1]. MERS coronavirus was transmitted through 27 countries and infected more than 2400 people beginning in 2012[2]. The COVID-19 pandemic has spread to more than 200 countries or areas and transmitted to more than 20 million people in 2020[3].

It is indisputable that infectious diseases easily spread through the world carried by tourists and frequent commercial air travel. Even if airports implement body temperature monitoring or inspection, blocking carriers who are asymptomatic remains difficult. Because of extensive business ties and large volume of travel between Taiwan and China, a pandemic occurring in China would be expected to spread to Taiwan very early and easily. The first recorded SARS case occurred in China in mid-November 2002. The first case in Taiwan was reported in early February 2003. COVID-19 was first noted in China in December 2019. The first confirmed case of COVID-19 in Taiwan was on January 21, 2020, carried by a Taiwanese woman who had been working in Wuhan, China. The speed of the spread of contagious disease from China to Taiwan became faster and faster. In addition, COVID-19 is known to be transmittable before the onset of symptoms, and most people do not have fever at the beginning of the disease[4,5,6]. The number of tourists traveling between China and Taiwan before the outbreak of COVID-19 was greater than that at the time before the outbreak of SARS. Therefore, researchers had predicted that Taiwan would be one of the countries most affected by COVID-19[7].

Patients choose to visit emergency departments (EDs) when they first develop epidemic-like illnesses, or ask for screening when they are afraid of infection. In Taiwan, EDs not only provided 24/7 services but also had relatively good accessibility and short waiting times[8]. Therefore, EDs played a critical role on the frontline of infection control in Taiwan, especially in the early stages of the pandemic. Because asymptomatic carriers can visit any ED at any time, once an epidemic prevention breach occurs at EDs, the 
risk of a hospital or community outbreak is increased[9]. Even if the number of patients visiting EDs decreases during an outbreak, EDs are still in danger without adequate protective equipment, consistent prevention policies, and synchronized infection control measures (ICMs)[10].

Most papers have discussed the COVID-19 pandemic in terms of diagnosis, treatment, and ICMs. The differences in ED responses to SARS and COVID are relatively few. Taiwan was severely affected by early outbreaks of both SARS and COVID-19. After the painful experience of SARS, Taiwan made many changes to its anti-epidemic policies. However, whether these reforms are achieving their aims has not been surveyed. The purpose of this study was to compare ED responses to the SARS and COVID-19 pandemics to survey the outcomes of these interventions. Analyzing the results of these changes may provide the insights into pandemic control for the benefit of the global community's preparedness and response to future pandemics.

\section{Methods}

Questionnaire results acquired from ED administrators during the SARS and COVID-19 pandemics were compared. The questionnaires were designed by ED experts after panel discussion and were approved by institutional review boards in 2003 and 2020. The overall contents of the questionnaires were similar and were designed to investigate epidemic prevention[11]. Data collection included hospital levels, type of personal protective equipment (PPE) provided, ICM types implemented, timing for using PPE and ICMs, and difficulty rating for ICM implementation.

Hospitals that provided 24/7 EDs were classified into 3 levels: medical centers (level A) with more than 500 hospital beds, regional hospitals (level B) with 200 to 499 beds, and local hospitals (level C) with fewer than 200 beds. Basic PPE consisted of head and shoe coverings, goggles, face shields, gloves, aprons, disposable gowns, surgical masks, and N95 face-piece respirators. Highgrade PPE included powered P100/N100/FFP3 air-purifying respirators and full-body isolation suits. ICMs were classified as basic and additional. The basic ICMs were recommended by the Taiwan Centers for Disease Control (CDC), and additional ICMs depended on the decisions of different hospital incident command systems. The epidemic period was divided into 2 stages. The early stage was defined as the time from the first infected case announcement to the occurrence of the first mortality case, and the late stage immediately followed the early stage. The difficulty of implementing ICMs at EDs was rated on a scale from mildly difficult (1 point) to very difficult (5 points).

The association between categorical variables was analyzed using chi-square and Fisher's exact tests. $P$ values of $<.05$ were considered significant. The difficulty rating scale for implementation of ICMs was represented by median values.

\section{Results}

In Taiwan, 213 EDs were open 24/7 in 2003, whereas in 2020, 200 EDs were open 24/7. One hundred EDs responded to the questionnaire (respondent rate $=46.9 \%$ ) in 2003, and 131 EDs responded in 2020 (respondent rate $=65.5 \%$ ). The increase in the respondent rate was mainly contributed by level B hospitals.

Comparisons of the PPE supplied at different levels of ED are presented in Table 1, revealing that most of the basic PPE supply was improved during COVID-19 compared with the SARS epidemic. This change was significant in level B and level C EDs. The supply of surgical masks and $\mathrm{N} 95$ masks was improved in level B EDs $(P<.001)$. Regarding high-grade PPE, the P100/N100/FFP3 supply was decreased in level A and B EDs during COVID-19. The supply of powered air-purifying respirators was increased at all levels of hospital during COVID-19, but the percentages of supplies were still low, particularly at level B (from $7 \%$ to $26 \%$ ) and C hospitals (from $7 \%$ to $21 \%$ ).

Comparisons of ICM implementation in different hospitals during SARS and COVID-19 are presented in Table 2. The implementation of basic ICMs was 100\% in level A EDs during the COVID-19 pandemic. The quarantine of fever patients outside EDs was significantly decreased in level $\mathrm{B}(P=.008)$ and $\mathrm{C}(P=.004) \mathrm{EDs}$. The percentage of fever patients quarantined in isolation rooms was also decreased in level B (from $86 \%$ to $69 \%$ ) and C (from $81 \%$ to $70 \%$ ) EDs. Regarding additional ICMs, the implementation of fever triage wards or restricted fever patient admission was significantly decreased in level B and C EDs during COVID-19. The measure of restricted patient transfer was significantly decreased in level C EDs (from $54 \%$ to $21 \%$ ). More level A 
EDs established screening teams $(P=.036)$, but level C EDs decreased their implementation of this ICM $(P=.004)$ during COVID-19. The ratio of ED closure was decreased at all levels, particularly in level B and C EDs $(P<.001)$ during COVID-19.

Table 3 displays the timing of responses at EDs by different hospital levels and types during the SARS and COVID-19 epidemics. With respect to the timing of ED responses, the timing for using PPE and implementing ICMs were all started significantly earlier for all types and levels of ED.

The rating scale for ICMs for all EDs is presented in Figure 1, and reveals that additional ICMs were relatively difficult to implement compared with basic ICMs in both SARS and COVID-19 groups, except for the institution of isolation rooms at EDs. Establishing a COVID-19 screening team and restricting patient transfers were less difficult than during the SARS epidemic. However, instituting a fever triage ward, restricting admission of patients with fever, and closing EDs became more difficult during COVID-19.

\section{Discussion}

In a closely interconnected world, poor responses to infectious disease can lead to severe adverse effects for public health, economic growth, tourism, business and industrial performance, and political and social stability. EDs are the frontlines of infectious disease in medical care systems and thus are key locations for epidemic prevention. This is the first paper comparing ED responses for SARS epidemic with those for COVID-19 epidemic. During the SARS outbreak in Taiwan, nearly $30 \%$ of cases were health care workers (HCWs), and this high infection rate resulted in 7 hospital outbreaks and lockdowns. During a 5-month period from early February to early July, there were 346 SARS cases and 73 (21.1\%) deaths[12]. In addition, some of these HCWs were sources of disease transmission. However, during 7 months of COVID-19 from late January to late August, there were 514 new cases and 7 deaths (1.4\%), of which $1 \%$ were HCWs; no hospitals were closed[13]. These differing results may be strongly associated with reforms to epidemic prevention policies after the SARS outbreak.

Changes in PPE preparedness

During the SARS epidemic, a lack of alertness to infectious disease and basic PPE usage resulted in nosocomial infection and community infections[14]. The Taiwan CDC learned from the SARS outbreak and reformed infection control policy stipulating that hospitals should prepare at least 1 month of basic PPE. In addition, a hospital accreditation system would audit their performance. The policy helped hospitals stay alert and maintain adequate basic PPE during normal periods. When the pandemic was announced, basic PPE could be put into practice immediately. During the COVID-19 epidemic, this policy change was reflected in the significant improvement in basic PPE use at all levels of ED, and especially in regional and local hospitals. However, preparing PPE was still difficult in some non-medical center hospitals. Furthermore, when the pandemic increased in severity and sustained, the supply of basic PPE such as surgical masks or N95 masks may have become short. The continuous supply of adequate basic PPE may have become a daunting challenge for all hospitals.

The need for high-grade PPE at EDs is still unclear. The coronavirus is predominantly transmitted by droplets but can become aerosolized during aerosol-generating procedures[15]. EDs are places where high-level precautions must be taken against airborne transmission because many critical patients require emergency intubation and respiratory therapies. A high level of respiratory protection (above the level of N95 masks) is recommended for aerosol-generating procedures[16]. In this study, the preparedness of high-grade PPE was increased at all levels of hospital during the COVID-19 pandemic, but the percentage was still low, especially in small- to medium-level hospitals. Even in medical centers with a high preparedness of high-grade PPE, there were difficulties coping with the large number of critical patients. This indicates that EDs in Taiwan still faced the risk of infection by airborne transmission. Emergency physicians have published some designs for isolation in the literature during the COVID-19 pandemic $[17,18]$. This phenomenon may reflect that the provision of high-grade PPE in Taiwan may actually be insufficient, such that physicians have needed to design their own devices or no-contact methods to protect themselves[19]. The hospitals and government should formulate a new policy to solve this problem as soon as possible.

Changes in ICM implementation

After the SARS outbreak, the Taiwan CDC changed some ICMs including encouraging the establishment of isolation room at EDs, recording TOCC (histories of travel, occupation, contact, and cluster) at ED triage, and providing masks free of charge for fever 
patients. Hospital infectious control teams were requested by the Taiwan Joint Commission on Hospital Accreditation to audit performance. When the epidemic prevention alert was activated, all patients with TOCC were requested to be treated outside of ED. Very early during the COVID-19 epidemic, the CDC ordered EDs to set up body temperature screening devices at entrances and to restrict visitors[20,21]. The performance and cooperation of hospitals were effective. However, there were still difficulties in implementing the quarantine of fever patients outside ED and establishing isolation rooms, especially in small- to medium-sized hospitals, during both the SARS and COVID-19 epidemics. The main reason may be the difficulty of modifying ED spaces that are also not sufficiently large to set up adequate isolation rooms. When there were patients who needed to lie in bed or required resuscitation, the problem of insufficient quarantine space became the largest challenge for EDs. Rapid polymerase chain reaction (PCR) testing may help to reduce demand; however, when many suspected patients are rushed into EDs or the PCR tests are not accurate or rapid enough, the prevention system may malfunction[22]. At such times, it may be recommended to transfer infected patients into mobile cabin hospitals or specialized hospitals[23].

The implementation of additional ICMs is dependent on the considerations of different hospital administrators. The implementation of separate fever triage wards and the restriction of admissions of fever patients were significantly decreased in small- to medium-sized hospitals during the COVID-19 epidemic, which may be because the COVID-19 virus was more quickly identified by PCR testing than was the SARS virus[24,25]. This could also explain the decreased percentages of inward restricted patient transfers and outward suspected case transfers. For the next pandemic, the earlier in time that pathogen information and identification is released and applied, the more effectively additional ICMs can be chosen at EDs.

Changes in response timing

During the SARS epidemic, ambiguous information caused hospitals to worry about the epidemic's effects on patient volume and revenue, such that announcements regarding PPE usage and implementation of ICMs became slow and hesitant. With similar reasoning to that of the hospital administrators, the government was concerned about social panic and economic consequences, such that many ICMs became controversial. Advice from experts was suppressed, such that orders for basic PPE and ICM implementation often began later than did the frontline HCWs themselves[26]. During the COVID-19 epidemic, not only was the Central Epidemic Command Center (CECC) quickly established, but their orders were also consistent and powerful. After the experience with SARS, the government provided bailout programs to the hospitals, and hospitals were more willing to cooperate with the government's epidemic prevention polices. Financial backup from the government also increased the authority of the CECC. During the COVID-19 epidemic, the ED responses at the early stages proved that effective commands from the CECC underwritten by financial support to hospitals from the government were key for epidemic prevention.

\section{Changes in ICM performance}

The effectiveness of epidemic prevention responses in EDs mainly depends upon the provision of sufficient resources at the hospital. The difficulties faced by EDs often reflect hospitals' administrative considerations and the availability of resources. The closure of EDs may be one of the most effective ways to prevent HCWs from burning out and hospitals collapsing during a pandemic[27,28]. However, this measure may collapse the medical care system, cause social panic, and violate patient rights, and thus it was not a sensible or smart measure for preventing hospital outbreak.

Although the CECC does not support restricting inward or outward transfers of suspected patients, when the resources of the hospital isolation room are insufficient, these emergency response methods may be used. After the SARS outbreak, the CDC supported many hospitals in different locations to establish qualified laboratories for reverse transcription-PCR testing, and hospitals could therefore identify infected patients more quickly. The use of the isolation rooms became more accurate and the pressure to set up a fever screening team or to restrict patient transfers was relatively reduced. This may explain why it was more difficult to restrict fever patient admissions than to implement fever screening wards. However, as mentioned, if too many infected patients were identified, EDs would be shut down immediately because of a lack of adequate isolation rooms.

\section{Conclusion}

Early, rapid, and effective responses to the COVID-19 epidemic at EDs prove that the policy reforms implemented after SARS have so far been effective in Taiwan. Adequate PPE preparedness, effective ICM implementation, financial support from the government, 
and a powerful CECC were key to preventing outbreaks and minimizing social disruptions during the pandemic. However, some hidden weaknesses were still present, including inadequate preparedness of high-grade PPE and incomplete implementation of ICMs in small- and medium-sized private hospitals. These may become the weak points for epidemic prevention in next wave or next pandemic.

\section{Limitations}

Our study has some limitations. First, some hospitals did not return questionnaires, perhaps because they did not meet the standards required by the CECC, which caused bias. Second, speculations and explanations regarding the underlying reasons in the results of the questionnaire may be imprecise.

\section{Declarations}

Availability of data and materials

Data available within the article or its supplementary materials.

Acknowledgements

We would like to thank our emergency department partners who volunteer for participation in this study.

Funding

Not applicable

Author information

Chia-Wei Lin ${ }^{1}$, Kuan-Ho Lin ${ }^{1}$, Hong-Mo Shih ${ }^{1}$, Kai-Wei Yang ${ }^{1}$, Kao-Pin Hwang ${ }^{2}$, Wei-Kung Chen ${ }^{1}$

${ }^{1}$ Emergency Department, China Medical University Hospital, Taiwan

${ }^{2}$ Division of Infectious Disease, China Medical University Hospital, Taiwan

\#First author:

Chia Wei Lin

*Correspondence:

Wei Kung Chen

Email: ercwk@mail.cmuh.org.tw

Affiliations

Emergency Department of China Medical University Hospital, No. 2, Xueshi Rd., North Dist., Taichung City 404, Taiwan

Contributions

WKC, CWL conceived the study design. KKL and KPH collected the study records and analyzed the data. HMS, CWL and KWY drafted the manuscript. All authors have read, edited, and approved the final manuscript.

Corresponding author

WKC is chief of staff of the emergency department of China Medical University Hospital, a medical center and teaching hospital in central Taiwan. His primary research interests include disaster response planning and emergency department management. 
Ethics declarations

Ethics approval and consent to participate

The protocol for the study has been reviewed and approved by China Medical University \& Hospital Research Ethics Center of Institutional Review Board (CMUH109-REC2-052).

Consent for publication

Not applicable.

Competing interests

The authors declare that they have no competing interests.

\section{References}

1. World Health Organization. SARS (Severe Acute Respiratory Syndrome). https://www.who.int/ith/diseases/sars/en/. Accessed 17 Oct 2020.

2. World Health Organization. Middle East respiratory syndrome coronavirus (MERS-CoV). https://www.who.int/emergencies/mers-cov/en/. Accessed 17 Oct 2020.

3. World Health Organization. WHO Coronavirus Disease (COVID-19) Dashboard. https://covid19.who.int/. Accessed 17 Oct 2020.

4. Richardson S, Hirsch JS, Narasimhan M, Crawford JM, McGinn T, Davidson KW, et al. Presenting Characteristics, Comorbidities, and Outcomes Among 5700 Patients Hospitalized With COVID-19 in the New York City Area. JAMA. 2020;323(20):2052-9. https://doi.org/10.1001/jama.2020.6775. PMID: 32320003.

5. Guan WJ, Ni ZY, Hu Y, Liang WH, Ou CQ, He JX, et al. Clinical Characteristics of Coronavirus Disease 2019 in China. N Engl J Med. 2020; 382(18):1708. https://doi.org/10.1056/nejmoa2002032. PMID: 32109013.

6. Chan JFW, Yuan S, Kok KH, To KKW, Chu H, Yang J, et al. A familial cluster of pneumonia associated with the 2019 novel coronavirus indicating person-to-person transmission: a study of a family cluster. Lancet. 2020; 395(10223):514-23. https://doi.org/10.1016/s0140-6736(20)30154-9. PMID: 31986261.

7. Gardner L. Modeling the Spreading Risk of 2019-nCoV. Johns Hopkins Whiting School of Engineering. https://systems.jhu.edu/research/public-health/ncov-model-2. Accessed 17 Oct 2020.

8. Wu TY, Majeed A, Kuo KN. An overview of the healthcare system in Taiwan. London J Prim Care (Abingdon). 2010;(2):115-9. https://doi.org/10.1080/17571472.2010.11493315. PMID: 25949636.

9. Lau JT, Fung KS, Wong TW, Kim JH, Wong E, Chung S, et al. SARS transmission among hospital workers in Hong Kong. Emerg Infect Dis. 2004; 10(2):280-6. https://doi.org/10.3201/eid1002.030534. PMID: 15030698.

10. Kathleen PH, Aaron KP, Jourdan D. Michael AC. Impact of the COVID-19 Pandemic on Emergency Department Visits - United States, January 1, 2019-May 30, 2020. MMWR Morb Mortal Wkly Rep. 2020;69(23):699-704.

11. Chen WK, Wu HDI, Lin CC, Cheng YC. Emergency Department Response to SARS, Taiwan. Emerg Infect Dis. 2005;11(7):10671073.

12. Government of Taiwan. SARS and Flu Prevention: SARS Outbreak in Taiwan. https://www.cdc.gov.tw/InfectionReport/Info/SVtdjRgESOT_EwbAhjlJ4g?infold=TjfRPEL5yuvaWGWFleyY9A. Accessed 17 Oct 2020.

13. Taiwan Center for Disease Control. Epidemic report in Taiwan. Ministry of Health and Welfare, Taiwn. https://covid19.nchc.org.tw/dt_005-covidTable_taiwan.php. Published September 2020. Accessed 17 Oct 2020.

14. Hsueh PR, Yang PC. SARS Outbreak in Taiwan. Emerg Infect Dis. 2004 ;10(8): 1514-1515.

15. World Health Organization. Infection prevention and control of epidemic- and pandemic-prone acute respiratory infections in health care. https://apps.who.int/iris/bitstream/handle/10665/112656/9789241507134_eng.pdf?sequence=1. Accessed 17 Oct 2020. 
16. Centers for Disease Control and Prevention. Interim Domestic Guidance on the Use of Respirators to Prevent Transmission of SARS. from Severe Acute Respiratory Syndrome (SARS). https://www.cdc.gov/sars/clinical/respirators.html. Published 2005. Accessed 17 Oct 2020.

17. Seak CJ, Liu YT, Ng CJ. Rapid responses in the emergency department of Linkou Chang Gung Memorial Hospital, Taiwan effectively prevent spread of COVID-19 among healthcare workers of emergency department during outbreak: Lessons learnt from SARS [Epub ahead of print]. Biomed J. 2020. doi:10.1016/j.bj.2020.06.002.

18. Lien WC, Wu JL, Tseng WP, Ko PC, Chen SY, Tsai MS. Fight COVID-19 Beyond the Borders: Emergency Department Patient Diversion in Taiwan. Ann Emerg Med. 2020;75(6):785-787.

19. Lin CH, Lee KI, Lee FY, Gan CCR, Tseng YC, Chen WK. Protection effectiveness of a building-integrated COVID-19 sampling station that uses a sealed acrylic window as a physical barrier. Ann Emerg Med. 2020;76(3):376-377.

20. Gan WH, Lim JW, Koh D. Preventing Intra-hospital Infection and Transmission of Coronavirus Disease 2019 in Health-care Workers. Saf Health Work. 2020; 11(2):241-243.

21. Chiu WT, Laporte RP, W J. Determinants of Taiwan's Early Containment of COVID-19 Incidence. AM J Publicc Health. 2020; 110(7):943-944.

22. Ramdas K, Darzi A, Jain S. 'Test, re-test, re-test': using inaccurate tests to greatly increase the accuracy of COVID-19 testing. Nat Med. 2020;12:1-2.

23. Yao W, Wang X, Liu T. Critical role of Wuhan cabin hospitals in controlling the local COVID-19 pandemic. Infect Control Hosp Epidemiol. 2020;22:1-2.

24. Taiwan Center for Disease. Adequate testing capacity and precisely locate potentially infected individuals. Ministry of Health and Welfare, Taiwn. https://covid19.mohw.gov.tw/en/cp-4788-53906-206.html. Published May 14, 2020. Updated July 24. Accessed 17 Oct 2020.

25. Ho MS, Su IJ. Preparing to prevent severe acute respiratory syndrome and other respiratory infections. Lancet Infect Dis. 2004;4(11):684-689.

26. Chen KT, Twu SJ, Chang HL, et al. SARS in Taiwan: an overview and lessons learned. INT J INFECT DIS. 2005;9(2):77-85.

27. McDonald LC, Simor AE, Su IJ, et al. SARS in Healthcare Facilities, Toronto and Taiwan. Emerg Infect Dis. 2004;10(5):777-781.

28. Shaw K. The 2003 SARS outbreak and its impact on infection control practices. Public Health. 2006;120(1):8-14.

\section{Tables}

TABLE 1. Comparisons of the PPE supplied at different levels of ED 


\begin{tabular}{|c|c|c|c|c|c|c|c|c|c|}
\hline \multirow{3}{*}{$\begin{array}{l}\text { Personal protection } \\
\text { equipment(PPE) }\end{array}$} & \multicolumn{2}{|c|}{ Hospital level A } & \multirow{3}{*}{$\begin{array}{l}\mathrm{p}- \\
\text { value }\end{array}$} & \multicolumn{2}{|c|}{ Hospital level B } & \multirow{3}{*}{$\begin{array}{l}\mathrm{p}- \\
\text { value }\end{array}$} & \multicolumn{2}{|c|}{ Hospital level C } & \multirow{3}{*}{$\begin{array}{l}\mathrm{p} \text { - } \\
\text { value }\end{array}$} \\
\hline & SARS & COVID19 & & SARS & COVID19 & & SARS & COVID19 & \\
\hline & $n=15(\%)$ & $\mathrm{n}=16(\%)$ & & $n=28(\%)$ & $\mathrm{n}=58(\%)$ & & $n=57(\%)$ & $n=57(\%)$ & \\
\hline \multicolumn{10}{|l|}{ Basic PPEs } \\
\hline Head or shoe cover & $13(87)$ & 16(100) & 0.225 & $25(89)$ & $58(100)$ & 0.011 & $52(91)$ & $57(100)$ & 0.022 \\
\hline Goggles & $12(80)$ & 16(100) & 0.101 & $21(75)$ & $57(98)$ & $<0.001$ & $46(81)$ & $56(98)$ & 0.002 \\
\hline Face shield & $15(100)$ & $16(100)$ & - & 25(89) & $58(100)$ & 0.011 & $47(82)$ & $57(100)$ & $<0.001$ \\
\hline Gloves & 14(93) & $16(100)$ & 0.293 & $24(86)$ & $58(100)$ & 0.003 & $55(96)$ & $57(100)$ & 0.153 \\
\hline Apron & $11(73)$ & $16(100)$ & 0.026 & $22(79)$ & $56(97)$ & 0.007 & $42(74)$ & $52(91)$ & 0.013 \\
\hline Disposable gown & $10(67)$ & $16(100)$ & 0.011 & $25(89)$ & $57(98)$ & 0.063 & $35(61)$ & $55(96)$ & $<0.001$ \\
\hline Surgical mask & $10(67)$ & 16(100) & 0.011 & $17(61)$ & $58(100)$ & $<0.001$ & $38(67)$ & $57(100)$ & $<0.001$ \\
\hline N95 respiratory mask & $12(80)$ & 16(100) & 0.059 & $23(82)$ & $58(100)$ & $<0.001$ & $56(98)$ & $55(96)$ & 0.558 \\
\hline \multicolumn{10}{|l|}{ High grade PPEs } \\
\hline P100/N100/FFP3 & $11(73)$ & $7(44)$ & 0.095 & $13(46)$ & $17(29)$ & 0.118 & $5(9)$ & $6(11)$ & 0.751 \\
\hline $\begin{array}{l}\text { Powered air-purifying } \\
\text { respirator }\end{array}$ & $6(40)$ & $11(69)$ & 0.108 & $2(7)$ & $15(26)$ & 0.041 & $4(7)$ & $12(21)$ & 0.031 \\
\hline
\end{tabular}

TABLE 2. Comparisons of ICM implementation in different hospitals 


\begin{tabular}{|c|c|c|c|c|c|c|c|c|c|}
\hline \multirow{3}{*}{$\begin{array}{l}\text { Infection control } \\
\text { measure(ICM) }\end{array}$} & \multicolumn{2}{|c|}{ Hospital level A } & \multirow{3}{*}{$\begin{array}{l}\mathrm{p}- \\
\text { value }\end{array}$} & \multicolumn{2}{|c|}{ Hospital level B } & \multirow{3}{*}{$\begin{array}{l}\mathrm{p}- \\
\text { value }\end{array}$} & \multicolumn{2}{|c|}{ Hospital level C } & \multirow{3}{*}{$\begin{array}{l}\mathrm{p}- \\
\text { value }\end{array}$} \\
\hline & SARS & CoVID19 & & SARS & COVID19 & & SARS & COVID19 & \\
\hline & $\mathrm{n}=15(\%)$ & $n=16(\%)$ & & $\begin{array}{l}\mathrm{n}=28 \\
(\%)\end{array}$ & $\mathrm{n}=58(\%)$ & & $\begin{array}{l}n=57 \\
(\%)\end{array}$ & $\mathrm{n}=57(\%)$ & \\
\hline \multicolumn{10}{|l|}{ Basic ICMs } \\
\hline $\begin{array}{l}\text { Entrance body } \\
\text { temperature screen }\end{array}$ & $15(100)$ & $16(100)$ & - & $28(100)$ & $58(100)$ & - & $57(100)$ & $57(100)$ & - \\
\hline Visitors restriction & 14(93) & $16(100)$ & 0.483 & $24(86)$ & $58(100)$ & 0.003 & $55(96)$ & $53(93)$ & 0.401 \\
\hline $\begin{array}{l}\text { Quarantine of fever } \\
\text { patients outside of EDs }\end{array}$ & 14(93) & $16(100)$ & 0.483 & $28(100)$ & $45(78)$ & 0.008 & $56(98)$ & $47(82)$ & 0.004 \\
\hline $\begin{array}{l}\text { Quarantine fever patients } \\
\text { in isolation room }\end{array}$ & $15(100)$ & 16(100) & - & $24(86)$ & $40(69)$ & 0.095 & $46(81)$ & $40(70)$ & 0.191 \\
\hline \multicolumn{10}{|l|}{ Additional ICMs } \\
\hline $\begin{array}{l}\text { Instituted fever triage } \\
\text { ward }\end{array}$ & 11(73) & $14(88)$ & 0.394 & $22(79)$ & $29(50)$ & 0.011 & $38(67)$ & $21(37)$ & 0.001 \\
\hline $\begin{array}{l}\text { Restricted fever patient } \\
\text { admission }\end{array}$ & 13(87) & $9(56)$ & 0.062 & $21(75)$ & $17(29)$ & $<0.001$ & $52(91)$ & $12(21)$ & $<0.001$ \\
\hline $\begin{array}{l}\text { Restricted patient transfer } \\
\text { in }\end{array}$ & $5(33)$ & $4(25)$ & 0.609 & $7(25)$ & $11(19)$ & 0.519 & $31(54)$ & $16(28)$ & 0.004 \\
\hline $\begin{array}{l}\text { Suspected case-patients } \\
\text { transfer out }\end{array}$ & $4(27)$ & $2(13)$ & 0.318 & $9(32)$ & $20(34)$ & 0.829 & $43(75)$ & $33(58)$ & 0.046 \\
\hline Screening team & $8(53)$ & $14(88)$ & 0.036 & $13(46)$ & $32(55)$ & 0.446 & $40(70)$ & $25(44)$ & 0.004 \\
\hline Closure of ED & $3(20)$ & $1(6)$ & 0.253 & $5(18)$ & $0(0)$ & $<0.001$ & $15(26)$ & $0(0)$ & $<0.001$ \\
\hline
\end{tabular}

TABLE 3. Comparison of the response timing at EDs by different hospital levels and types

\begin{tabular}{|c|c|c|c|c|c|c|c|c|c|c|}
\hline \multirow[t]{3}{*}{ Hospital } & \multicolumn{4}{|c|}{ Timing of PPEs } & \multirow{3}{*}{$\begin{array}{l}P \\
\text { value }\end{array}$} & \multicolumn{4}{|c|}{ Timing of ICMs } & \multirow{3}{*}{$\begin{array}{l}\mathrm{P} \\
\text { value }\end{array}$} \\
\hline & \multicolumn{2}{|c|}{ SARS $(n=100)$} & \multicolumn{2}{|c|}{$\operatorname{COVID}(n=131)$} & & \multicolumn{2}{|c|}{ SARS $(n=100)$} & \multicolumn{2}{|c|}{$\operatorname{COVID}(n=131)$} & \\
\hline & Early(\%) & Late(\%) & Early(\%) & Late(\%) & & Early(\%) & Late(\%) & Early(\%) & Late(\%) & \\
\hline \multicolumn{11}{|l|}{ Level } \\
\hline$A$ & $4(4)$ & $11(11)$ & $16(12)$ & $0(0)$ & $<0.001$ & $3(3)$ & $12(12)$ & $15(12)$ & $1(1)$ & $<0.001$ \\
\hline B & $2(2)$ & $26(26)$ & $47(36)$ & $11(8)$ & $<0.001$ & $8(8)$ & $20(20)$ & $46(35)$ & $12(9)$ & $<0.001$ \\
\hline C & $14(14)$ & $43(43)$ & $49(38)$ & $8(6)$ & $<0.001$ & $24(24)$ & 33(33) & $36(27)$ & $21(16)$ & $<0.039$ \\
\hline \multicolumn{11}{|l|}{ Type } \\
\hline Public & $2(2)$ & $28(28)$ & $46(35)$ & $6(5)$ & $<0.001$ & $10(10)$ & $20(20)$ & $46(35)$ & $6(5)$ & $<0.001$ \\
\hline Private & $18(18)$ & $52(52)$ & $66(50)$ & $13(10)$ & $<0.001$ & $25(25)$ & $45(45)$ & $57(43)$ & $22(17)$ & $<0.001$ \\
\hline
\end{tabular}

\section{Figures}




\section{Rating scale of ICM implementation (median)}

Basic ICM

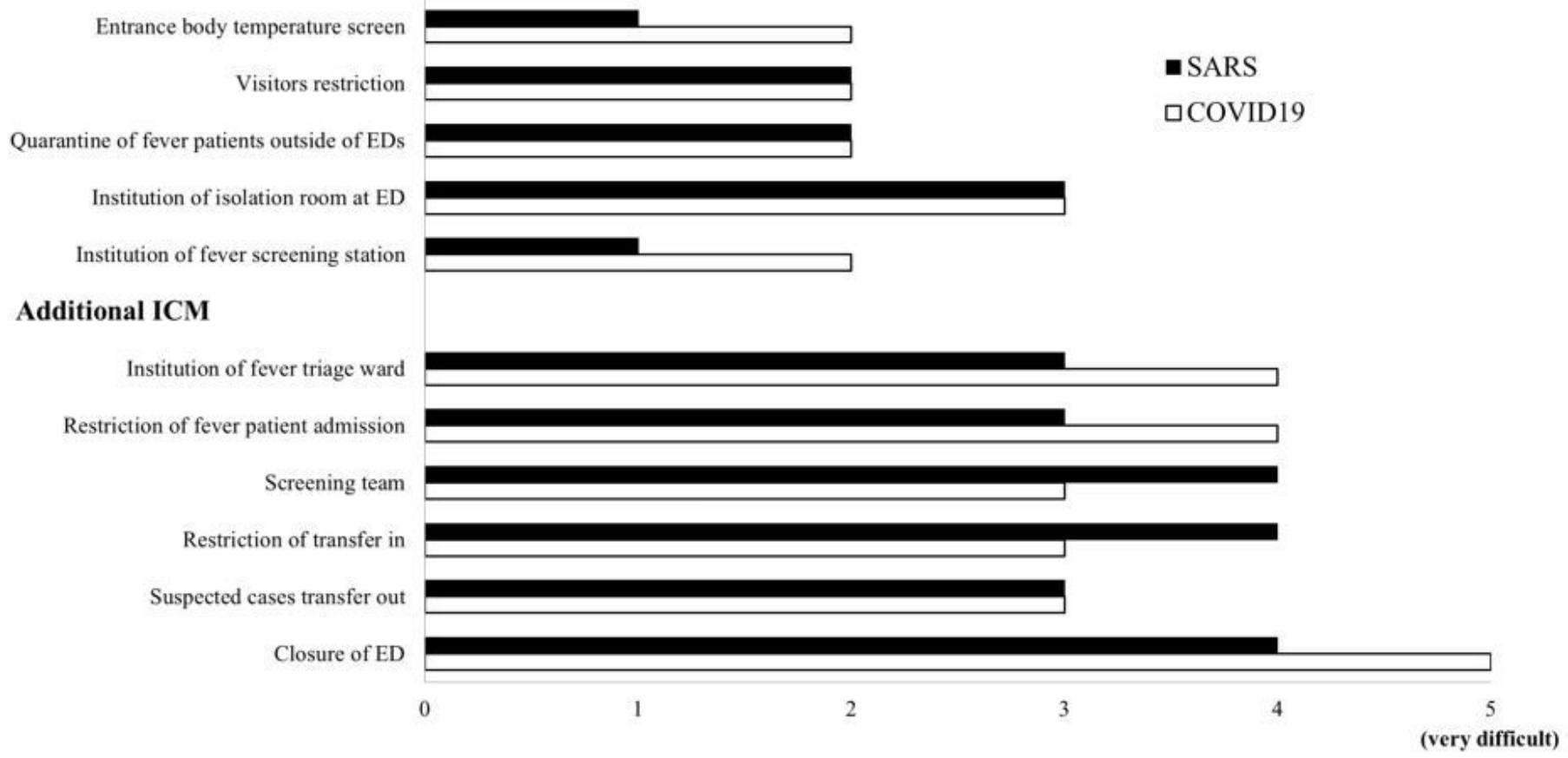

Figure 1

Rating Sclae of ICM implementation (median) 


\section{Rating scale of ICM implementation (median)}

Basic ICM

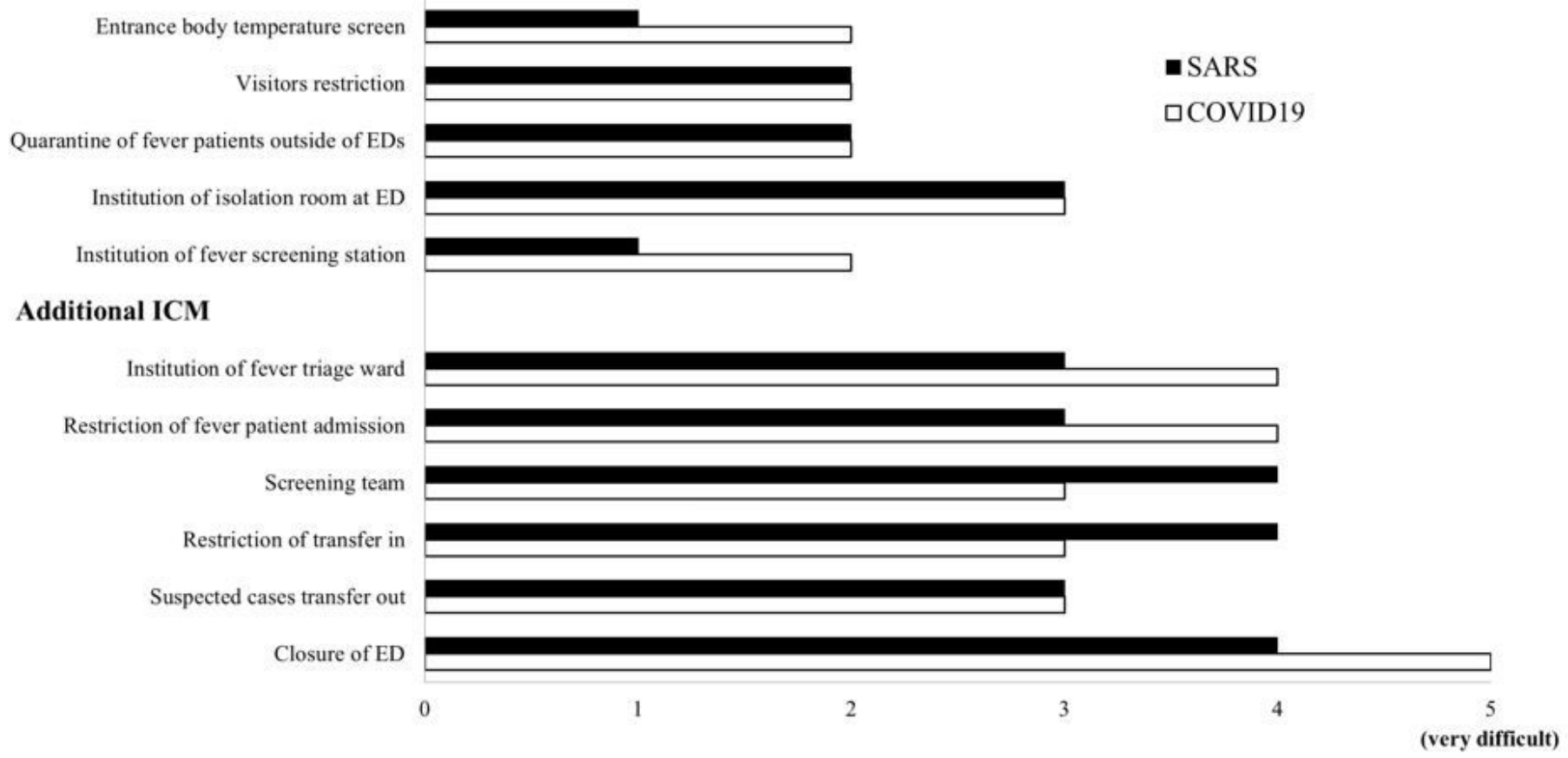

Figure 1

Rating Sclae of ICM implementation (median) 


\section{Rating scale of ICM implementation (median)}

Basic ICM

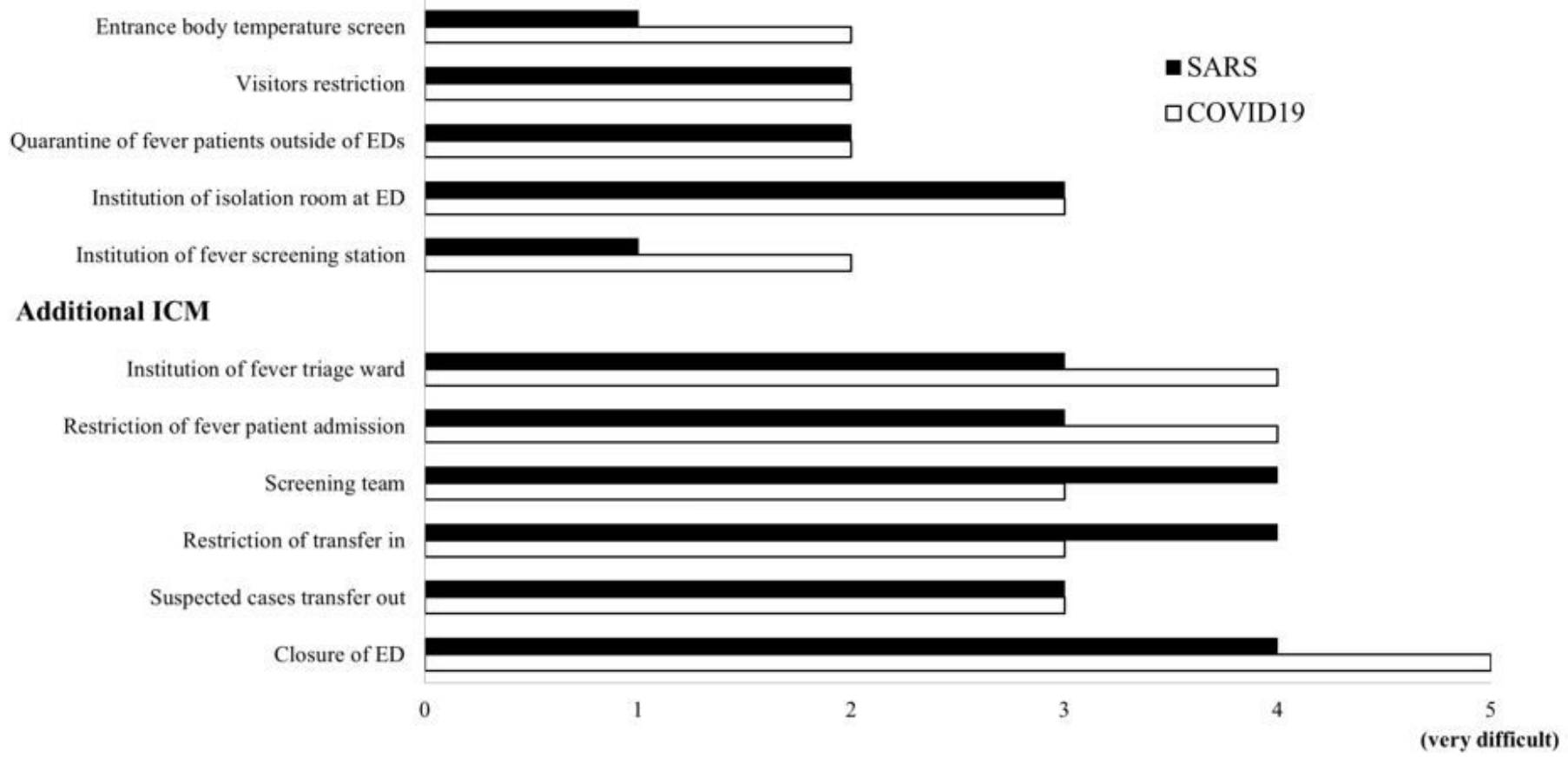

Figure 1

Rating Sclae of ICM implementation (median) 